\title{
Conocimiento profesional docente: conocimiento académico, saber experiencial, rutinas y saber tácito
}

\section{Professional teaching knowledge: academic knowledge, practical knowledge, routines and tacit knowledge}

\author{
Lic. Patricia Belén Demuth Mercado ${ }^{1}$
}

Fecha de recepción: febrero 2011

Fecha de aceptación y versión final: junio 2011

Resumen: El presente artículo expone resultados de una investigación realizada con docentes del nivel Polimodal de la ciudad de Corrientes, de diferentes áreas disciplinares: Historia, Matemáticas, Biología y Filosofía.

Se encuadra en la línea de investigación didáctica sobre el conocimiento profesional docente, su construcción y evolución. En el trabajo se describen y analizan los discursos docentes en relación con la construcción de su conocimiento académico, los saberes basados en la experiencia profesional y las rutinas y guiones de acción que los docentes pudieron identificar, a partir de estos análisis se reflexiona sobre algunas teorías implícitas identificadas.

Los resultados muestran que los docentes ubican en primer lugar de importancia para la construcción del conocimiento profesional docente a la práctica docente (saber experiencial); en segundo lugar al contexto institucional a su vez atravesado por las diferentes problemáticas socioculturales y económicas; y en tercer lugar identifican a la formación inicial, diferenciando en ella la formación disciplinar respecto de la pedagógica. A esta última, le atribuyen una escasa significatividad para la resolución de problemas que la realidad les presenta.

Palabras claves: Didáctica, Conocimiento profesional docente, formación inicial, práctica docente.

Abstract: This article provides results of an investigation with teachers of the "Polimodal" level from the city of Corrientes city, from different subjects : History, Mathematics, Biology and Philosophy.

It belongs to the research programme: "professional teacher knowledge", its construction and development. This paper describes and analyses teachers speeches in connection with the construction of its academic knowledge, the knowledge based on the professional experience and the routines and scripts of action that teachers could identify, from these analyses we reflect on some implicit theories identified among teachers.

Results show that teachers located teaching practice (experiential knowledge) in first place of importance for the construction of professional teacher knowledge; secondly to the institutional context; and in third place identify teacher education, making difference between the disciplinary education and the pedagogical one. Teachers confer to this last one a little significance for the problems of the educative reality.

Key words: Pedagogy, Professional teacher knowledge, teacher education, teaching practice.

\footnotetext{
${ }^{1}$ Facultad de Humanidades - UNNE - correo electrónico: patriciademuth@ hotmail.com
} 


\section{Introducción}

Este trabajo forma parte de una serie de investigaciones que desde el año 2001 se vienen realizando desde la cátedra de Didáctica de la Universidad Nacional del Nordeste- Argentina, y que actualmente se enmarcan en la línea de investigación que lleva adelante el Grupo CyFOD, "Conocimiento y Formación Docente", sobre este conocimiento profesional.

El equipo de investigación está compuesto por docentes de las cátedras de Didáctica I y Psicología del Aprendizaje del profesorado y licenciatura en Ciencias de la Educación, y Didáctica II del profesorado en Letras de la Facultad de Humanidades; y de Didáctica de los profesorados en Química y Física de la Facultad de Ciencias Exactas, Naturales y Agrimensura de la UNNE.

En las investigaciones precedentes se describieron, analizaron, reconstruyeron y compararon diferentes concepciones epistemológicas, de enseñanza y de aprendizaje, y su relación con la práctica docente; pertenecientes al conocimiento profesional de profesores en ejercicio y futuros docentes del nivel universitario, no universitario y nivel Polimodal.

Los resultados que a lo largo de los últimos años se fueron obteniendo como equipo de investigación respecto de las características principales que poseen las concepciones epistemológicas y didácticas, se orientan a concepciones y prácticas tradicionales-tecnicistas de enseñanza y aprendizaje, con visiones cercanas a paradigmas epistemológicos absolutistas. (Alcalá, M. T., 2002; Demuth, P. y Alcalá, M. T., 2005; Demuth, P. , Fernández, G. y Alcalá M. T., 2006; Fernández, G., Demuth, P. y Viña, N., 2007; Fernández, G., Acevedo, N. y Demuth, P. , 2007; Alcalá, M. T., Fernández G. y Demuth, P., 2008)

Dichos resultados mantienen relación con los presentados por Porlán y Rivero (1998), quienes exponen diferentes trabajos de investigación en torno a la temática. Los autores reconocen en las concepciones tradicionales y tecnicistas de enseñanza y aprendizaje, y en el paradigma epistemológico absolutista (con orientación empirista, principalmente) las características centrales del conocimiento profesional dominante del profesorado en los diferentes niveles del sistema educativo español.

\section{Orígenes y aportes referenciales fundamentales}

Abordar la problemática del conocimiento profesional docente, implica iniciar este recorrido desde el reconocimiento de los orígenes de este programa de indagación. A lo largo de los años, diferentes autores fueron realizando tentativas de sistematización del programa, que fueron retomadas en numerosas investigaciones del mundo académico anglosajón y de manera creciente en programas y grupos de investigación españoles. En esta oportunidad, retomaremos los aportes, a nuestro modo de ver, más significativos al momento de desentrañar este recorrido.

Una de las primeras sistematizaciones fue la realizada por Carter (1990) quien nos planteaba que el modelo "Teacher Thinking", denominación originaria para el estudio del profesor, sus pensamientos y sus conocimientos, ha seguido tres líneas de análisis:

1.) estudios sobre el procesamiento de la información y comparación entre profesores expertos-principiantes

2.) estudios sobre el conocimiento práctico, incluyendo conocimiento personal y conocimiento ecológico del aula 


\section{3.) estudios sobre conocimiento didáctico del contenido}

Una segunda sistematización a mencionar, es la realizada por Fenstenmacher (1994), en la cual examina diversos programas de investigación que se desarrollan en torno al conocimiento del profesor o sobre la enseñanza.

En su segundo capítulo, realiza una interesante descripción de la literatura sobre el conocimiento del profesor. La organización del capítulo se basa en cuatro preguntas guías: ¿Qué se sabe sobre la enseñanza eficaz?, ¿Qué saben los profesores?, ¿Qué conocimiento es esencial para enseñar?, ¿Quién produce conocimiento sobre la enseñanza?

En la primera pregunta distingue el concepto "conocimiento formal", ligado a los estudios del proceso-producto, en la segunda, relaciona aquellas investigaciones que intentan comprender lo que conocen los profesores a partir experiencia profesional (conocimiento práctico, práctico personal, situado, local, relacional, y tácito). A estos tipos de conocimiento los reúne bajo la categoría de "conocimiento práctico del profesor" y menciona fundamentalmente a dos de los trabajos centrales: "conocimiento práctico personal" de Connelly y de Clandinin y a la "práctica reflexiva" de Schön.

El aporte fundamental en esta obra es la organización antes nombrada que permite reconocer dos tipos de conocimiento epistemológicamente diferenciados: el conocimiento formal y el conocimiento práctico. El autor comenta de manera clara que todas aquellas denominaciones que adjetivan el conocimiento: conocimiento estratégico, conocimiento proposicional, conocimiento relacional, conocimiento artesanal, conocimiento local, conocimiento del caso, conocimiento situado, conocimiento tácito, conocimiento personal, etc., no están mencionando necesariamente diversos tipos de conocimiento y en consecuencia restringe la denominación a dos categorías epistemológicas discretas: Formal y Práctica, y a partir de ellas va describiendo y reflexionando sobre los aportes más significativos de los programas de investigación que a su interior se desarrollan.

La tercera sistematización escogida es la realizada por el autor español Bolívar (1995), quien menciona que la investigación sobre el conocimiento de los profesores se puede dividir en dos grandes categorías: conocimiento práctico y conocimiento didáctico del contenido. Sostiene, a su vez que desde esta última línea de indagación se plantearon diferentes críticas a los programas sobre "conocimiento práctico", la limitación a procesos idiosincráticos pero a la vez genéricos, olvidando el conocimiento del contenido de la enseñanza.

Nos interesa profundizar en estas dos categorías establecidas por Bolívar ya que constituyen aproximaciones cercanas al planteo de éste trabajo. Dentro de la primera categoría que aborda el "conocimiento práctico" del profesor, mencionaremos a Donald Schön (1987) y a su propuesta de una nueva epistemología de la práctica.

En su obra alude la teoría de la indagación de Dewey y critica de manera contundente a aquella investigación universitaria que otorga status privilegiado al conocimiento sistemático, preferiblemente de carácter científico. Reconoce que en estos trabajos se desarrolla una idea dominante del conocimiento profesional riguroso, fundamentado en la racionalidad técnica, dejando fuera de ésta racionalidad y sin definición a aquellas zonas de la práctica 'cenagosas'. (Cf. Ibíd: 17)

Para el autor las situaciones problemáticas de la práctica son muy diferentes a las planteadas por esa racionalidad técnica, desde la cual "por lo general cuando más se aproxima uno a las ciencias básicas mas alto resulta su status académico. El conocimiento general, teórico y proposicional, disfruta de una posición privilegiada." ( Ibíd: 22) 
En oposición a esta racionalidad la epistemología de la práctica atiende a aquellas "zonas indeterminadas de la práctica - tal es el caso de la incertidumbre, la singularidad y el conflicto de valores- que escapan a los cánones de racionalidad técnica" ( Ibíd: 20). Desde esta epistemología la práctica profesional es comprendida como reflexión en la acción, desde una concepción constructivista en la cual el profesional construye aquellas situaciones de su práctica.

La segunda categoría tiene sus inicios en los discursos y las obras de Lee Shulman, y su interés fundamental por el conocimiento didáctico del contenido (Pedagogical Content Knowledge). En su artículo "Knowledge and Teaching: Foundations of the New Reform", Shulman (1987) menciona las escasas descripciones o análisis de docentes en las que se preste atención al manejo de las ideas del profesor en el aula. Este interés por la gestión del conocimiento guía la reflexión en torno a la pregunta sobre cuáles son las creencias, las concepciones y los conocimientos prácticos que le permiten a los profesores a enseñar como lo hacen.

Se interesa por presentar la base intelectual, práctica y normativa para la profesionalización de la docencia, en relación al contenido, el carácter y las fuentes de un conocimiento base para la enseñanza.

Sus planteos de análisis se orientan hacia la identificación y conceptualización de las fuentes del conocimiento base para la enseñanza y las implicaciones para las políticas formativas docentes, desde la concepción de que este conocimiento base para la enseñanza no tiene un carácter fijo y definitivo, en el cual se hace hincapié en la docencia como un acto de comprensión y razonamiento, de transformación y reflexión.

En esta misma dirección una de las principales discípulas de Shulman, Pamela Grossman, en el libro "The making of a teacher. Teacher knowledge and teacher education"

Reflexiona sobre las demandas que la realidad le estaba realizando a la investigación sobre el docente y la enseñanza, y menciona como una de las causas a la escasa evidencia sobre el contenido de educación de profesores y su influencia en el desarrollo del conocimiento profesional y sus creencias y por ende, a la reducida conceptualización existente entre el conocimiento profesional y formación en enseñanza. Por esta razón la pregunta acerca del conocimiento profesional de base para la enseñanza ha provocado la necesidad de impulsar estudios sobre la naturaleza del conocimiento necesario para la enseñanza y las fuentes de ese conocimiento. (Grossman, 1990: 9).

Una vez realizada la presentación de esta línea de indagación, podemos afirmar que la misma se fue construyendo a lo largo de los años, teniendo su inicio más claro en la segunda mitad de la década de los 80 , y se ha convertido en la actualidad en una de las principales corrientes de investigación en Didáctica.

Una representante del pensamiento español es la especialista Lourdes Montero (2001: 7) quien reflexiona acerca del estudio sobre el proceso de construcción de la indagación sobre el conocimiento profesional docente ya que "es una dirección emergente que permitiría progresar en la comprensión de los dominios del conocimiento de la enseñanza y las direcciones en que los profesores los adquieren" $y$ son estos progresos comprensivos sobre los conocimientos de la práctica educativa los que definen el desarrollo científico de la disciplina (Ibíd.: 16).

Porlán y Rivero (1998: 97) afirman que los profesores no son fácilmente permeables a las propuestas y reflexiones de los investigadores, dado que tienen concepciones implícitas y explícitas sobre las diferentes variables relacionadas con la 
enseñanza y que dichas concepciones no cambian automáticamente cuando se enfrentan a perspectivas diferentes.

Por estos motivos, es que se vuelve cada vez más necesaria la indagación de los procesos de pensamiento, las concepciones científicas, didácticas y curriculares que conforman su conocimiento profesional, desde los mismos docentes, para que su comprensión llegue fehacientemente a todos los sujetos educativos, en especial, claro está, a los profesores, entendidos como únicos sujetos que pueden hacer evolucionar el modelo de enseñanza predominante.(lbíd. : 98)

Junto con Montero (2001:134) se señala que el estudio realizado intenta incidir en la mejor comprensión del conocimiento profesional de los profesores, en dar explicación de su adquisición y, de esa manera, contribuir a repensar la función docente y la formación del profesorado. Sin desconocer que el aumento en el interés por el conocimiento de los profesores, si bien se encuentra motivado por factores prácticos, también lo motivan factores políticos y académicos.

\section{Algunas aproximaciones a la noción "Conocimiento profesional"}

Diferentes autores fueron caracterizando al conocimiento docente, estas caracterizaciones mantienen algunas consideraciones comunes y también resaltan rasgos distintivos. A continuación hemos seleccionado aquellas definiciones que se aproximan a nuestra propia manera de entender el conocimiento de los profesores.

Gimeno Sacristán afirma que el profesor posee significados adquiridos explícitamente durante su formación y también otros que son resultado de experiencias continuadas y difusas sobre los más variados aspectos que podamos distinguir en un curriculum: contenidos, destrezas, orientaciones metodológicas, pautas de evaluación (1997:212).

En esta línea, Rodrigo y otros (1993) sostienen que el conocimiento del profesor se trataría de un conocimiento pragmático que responde a demandas prácticas. En igual sentido Montero (2001: 129) asevera que corresponde a un tipo de conocimiento praxeológico, construido en contextos en los que se ejerce la práctica. Los docentes someterían a análisis crítico las teorías que sostienen, las prácticas en las que están insertos y los contextos sociales en los que se desenvuelven.

El autor español Angulo Rasco (1999: 306) desarrolla diferentes tipos de conocimiento docente y los corresponde bajo la categoría de conocimiento práctico como conocimiento fruto de la relación entre la formación teórica del docente y la interacción con el medio escolar. En tanto conocimiento práctico, no se encuentra separado del conocimiento teórico adquirido previamente, sino que, en realidad, se muestra como un conocimiento transformado en razón de la acción práctica del docente, de su experiencia personal. Ese conocimiento transformado en la acción práctica es el que constituye para el autor el conocimiento de oficio en el docente.

Elliott (1990) reconoce además, que el conocimiento profesional consiste en teorías prácticas o en marcos conceptuales -categorizaciones de problemas prácticos, sus explicaciones y soluciones- que subyacen a las prácticas profesionales. En el mismo sentido se afirma que el conocimiento profesional es un tipo de conocimiento experiencial, representado en imágenes o constructos más o menos esquemáticos y de carácter subjetivo, personal y situacional" (Marrero, 1993)

Por otro lado, en su artículo 'Knowledge and Teaching' del 2002, Kennedy retoma la teoría de Huberman (1983) sobre el conocimiento docente como conocimiento artesanal, en gran parte idiosincrásico y no-teórico. Huberman critica a la 
investigación sobre la naturaleza del conocimiento de los profesores, el gran interés teórico y el escaso interés práctico que predomina en ella. Por esta razón Kennedy propone que se distingan tres fuentes del conocimiento docente relevantes a la enseñanza para ser estudiadas en estas investigaciones: el conocimiento artesanal, adquirido a través de la experiencia, no-teórico e idiosincrásico; el conocimiento sistemático que se adquiere principalmente a través de las universidades y de las diferentes instituciones de formación, los artículos de la investigación, los diarios y las asociaciones profesionales, más teórico y organizado; y el conocimiento prescriptivo o normativo, que se adquiere generalmente a través de las políticas institucionales, más codificado que el conocimiento artesanal pero menos teórico que el conocimiento sistemático. (2002: 355-356)

Como se puede apreciar, las diferentes definiciones del conocimiento profesional docente apuntan a reconocer la presencia de un conocimiento formal y sistemático, construido en las instituciones de formación profesional, un conocimiento normativo fruto del deber hacer en los contextos de intervención profesional, pero mayoritariamente hacen hincapié en el conocimiento docente experiencial, pragmático, praxeológico, práctico o artesanal.

A partir de este desarrollo se entiende al Conocimiento Profesional como conocimiento práctico, epistemológicamente diferenciado y radicalmente distinto al que mayoritariamente existe, cuya construcción ha de ser gradual y progresiva, tomando en consideración las concepciones de partida de los profesores, sus obstáculos y sus posibles hipótesis de progresión que faciliten su evolución. (Porlán y Rivero: 1998: 10 y ss)

Considerando estas características se vuelve necesario retomar las conclusiones expuestas por Porlán y Rivero (Op.Cit. p. 60-64) a partir de una serie de estudios realizados que manifiestan cuál es el conocimiento profesional dominante, y cómo está compuesto para $\mathrm{n}$ avanzar desde esos datos en el estudio del conocimiento profesional con los docentes seleccionados para el trabajo de campo.

Los autores sostienen, en primera instancia que todo conocimiento profesional posee cuatro aspectos o tipos de conocimientos: conocimiento académico, saberes basados en la experiencia, rutinas y guiones de acción, conocimiento tácito; estos aspectos desarrollados a continuación, serán trabajados con los docentes seleccionados del estudio a fín de caracterizar su conocimiento docente,

- Conocimiento académico: conjunto de concepciones disciplinares, relativas al currículo o a las ciencias de la educación, saberes que se generan en el proceso de formación inicial. Son explícitos y están organizados, atendiendo a la lógica disciplinar, en el mejor de los casos.

- Saberes basados en la experiencia: Se refieren al conjunto de ideas concientes que los profesores desarrollan durante el ejercicio de la profesión acerca de diferentes aspectos de los procesos de enseñanza-aprendizaje.

Se manifiestan como creencias, como principios de actuación, metáforas, imágenes de conocimiento personal, orientan la conducta profesional. No poseen un alto grado de organización interna, pertenecen al conocimiento del "sentido común". Es adaptativo, con contradicciones internas, impregnado de valoraciones morales e ideológicas.

Según la investigadora argentina Alliaud (1998) estos saberes que denomina "de oficio" se tratan de conocimientos "personales" que surgen de circunstancias, acciones y experiencias que tuvieron contenido afectivo o relevancia emocional para el 
individuo. Sin embargo menciona que a la vez de ser personales poseen elementos comunes e integran la "cultura del trabajo de la enseñanza" como conjunto de supuestos básicos (actitudes, valores, creencias y formas de hacer las cosas), pautas de relación e interacción (entre los sujetos involucrados y también con el conocimiento) que son compartidos por los docentes.

Retoma la noción de "habitus" de Bourdieu para señalar que frente a los imprevistos el profesor reacciona con modelos más adaptados a lo largo de la experiencia.

- Rutinas y guiones de acción: Conjunto de esquemas tácitos que predicen el curso de los acontecimientos en el aula que contienen pautas de actuación concretas y estandarizadas para abordarlos.

Se organizan en el ámbito de lo concreto y en contextos muy específicos y se dan por impregnación ambiental.

Otra manera de nombrar este tipo de conocimientos lo realiza la autora francesa Chartier (2000) bajo la denominación de "saberes ordinarios" (sin sentido peyorativo), son saberes que orientan la acción, en este caso del docente.

Para la autora la acción pedagógica en el aula se basa en esos saberes ordinarios que son invisibles para los propios maestros y profesores, y sobre los cuales les es imposible teorizar. Tienen un estatuto diferente al de los saberes elaborados que se transmiten en la formación inicial, un estatuto basado en diferentes formas de autorización y en otros ámbitos de circulación y de transmisión. Su autoridad es la autoridad de la práctica, de la experiencia, de lo establecido, de lo que da resultado. Su ámbito de circulación, el de la informalidad de las relaciones entre pares, el de la sala de profesores, el de los recreos, el de la transmisión de secretos del oficio, el de la autoridad establecida por el sistema de poder o de contrapoder en cada una de las instituciones. (Finocchio y Pinkasz, 2006)

Por su parte, Schön (1987: 35) hace mención a aquellos procesos no lógicos, juicios, decisiones y acciones eficientes que realizan los profesionales de un modo espontáneo, sin ser capaces de establecer las reglas o los procedimientos que siguen.

- Teorías implícitas: Se refieren más bien a un no saber que a un saber. Manifiestan las posibles relaciones entre pensar y actuar. Son un tipo de concepciones que sólo pueden ponerse en evidencia con la ayuda de otras personas, ya que no son teorizaciones concientes ni aprendizajes académicos que se han convertido de manera significativa en creencias y pautas de actuación concreta. Suelen guardar relación con estereotipos sociales dominantes.

A partir de lo anterior, el presente estudio plantea la descripción y análisis de los discursos docentes del nivel medio de la ciudad de Corrientes en relación con la construcción de su conocimiento académico, los saberes basados en la experiencia profesional y las rutinas y guiones de acción, e identificar aspectos teóricos, prácticos explícitos e implícitos que inciden en la evolución de tales conocimientos.

\section{Materiales y métodos}

Debido a que este estudio se desprende de uno mayor mencionado en la introducción nos parece necesario relatar brevemente el proceso metodológico seguido. 
Desde la metodología cualitativa de investigación se abordó el análisis de las respuestas de los docentes seleccionados en tres fases diferentes que se explicarán a continuación.

La primera selección de la muestra de docentes se realizó a partir de la identificación y autorización para el trabajo de dos instituciones de la ciudad, una de ellas correspondiente a una institución privada ubicada en el micro centro de la misma, y la otra corresponde a una institución pública periférica. Esta variación en el contexto institucional permitió realizar cruce de datos interesantes para la totalidad del trabajo. A su vez, se establecieron los siguientes criterios para la identificación de los docentes: pertenencia a las áreas del conocimiento seleccionadas, diversa antigüedad en la docencia y disponibilidad para el trabajo de campo.

En una primera fase se procedió a un cuestionario abierto autoadministrado, aplicado a la totalidad de la muestra (24 docentes), que permitió una aproximación a la temática planteada. Los interrogantes apuntaban a establecer concepciones didácticas: ligadas a la enseñanza, ligadas al aprendizaje y ligadas a la evaluación; concepciones epistemológicas: ligadas al conocimiento científico y a su metodología; y por último, se solicitaba un breve relato de la Práctica docente. En la segunda fase se seleccionó a un grupo menor de docentes (4 docentes). Dicha selección se basó en las respuestas que los docentes brindaron en el cuestionario, a partir de éstas se procedió a observaciones no participantes de clases, para la elaboración de una guía abierta tentativa que dio lugar a la tercera fase que incluyó diferentes entrevistas en profundidad.

Las tres fases planteadas se relacionaron siguiendo el criterio de recursividad, ya que cada una de ellas sirvió como insumo para la proyección de la siguiente.

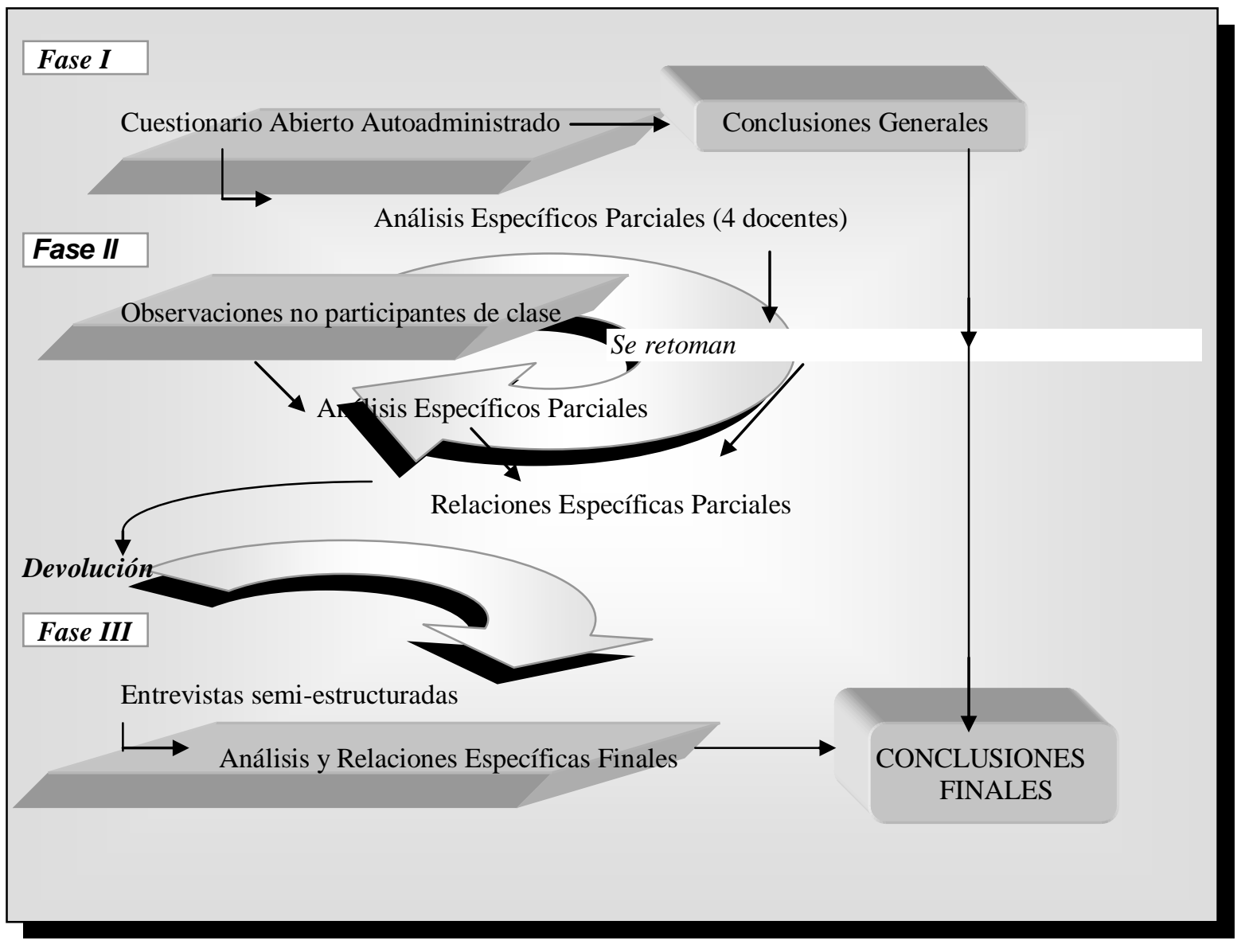


En el presente artículo se abordarán los análisis y resultados de la tercera fase planteada, que retoma en sí misma aspectos de las fases precedentes.

Como se mencionó anteriormente, las entrevistas en profundidad se realizaron con los 4 docentes del nivel, seleccionados a partir de los resultados del cuestionario abierto y una vez realizadas las observaciones.

En el diccionario de sociología de Fairchild (Sierra Bravo: 1994) se define la entrevista como " la obtención de información mediante una conversación de naturaleza profesional".

Esta definición comprende tanto la llamada entrevista estructurada formal o con cuestionario, como los diferentes tipos de entrevistas semiestructuradas o no estructuradas.

Se ha utilizado como instrumento de recolección esta segunda clase, donde además del objeto y el fin de la investigación, únicamente se indican los diversos puntos sobre los que interesa obtener información de los entrevistados. Por tanto, queda en todo caso a designio de entrevistador el número y el tipo de preguntas a realizar.

Teniendo como fundamento teórico a la definición que realizan Porlán y Rivero (1998: 10-11) sobre el Conocimiento Profesional Docente como conocimiento práctico, epistemológicamente diferenciado y radicalmente distinto al que mayoritariamente existe, cuya construcción ha de ser gradual y progresiva. $\mathrm{Y}$ entendiendo que su composición posee cuatro aspectos principales : Conocimiento académico, Saberes basados en la experiencia, Rutinas y guiones de acción, y Teorías implícitas

Se desagregan las diferentes temáticas a abordar en la entrevista

- Formación académica disciplinar. Inicial y de Perfeccionamiento

- Experiencia en el aula.

- Socialización con colegas de las instituciones a las que se pertenece

Posibles

Evoluciones

En función de las Concepciones de Enseñanza y Aprendizaje

Las entrevistas se iniciaron aclarando a cada docente que se iba a desarrollar un proceso recursivo y reflexivo.

Se expusieron en un primer momento una síntesis del encuadre teórico mencionado, y en segundo momento se realizó una devolución de las relaciones específicas parciales establecidas a partir de lo que los mismos docentes respondieron en la encuesta y lo registrado e interpretado en las observaciones.

Una vez desarrollado lo anterior se propuso a las docentes que comenten las impresiones generales que se les suscitaron al escuchar la devolución, y si deseaban agregar, preguntar o discutir algo respecto de lo dicho.

En función de lo que los docentes fueron desarrollando, se trabajaron sobre las tres temáticas antes mencionadas, intentado la construcción de relaciones entre estas temáticas y sus concepciones y conocimientos profesionales. 


\section{Discusión de resultados}

Para caracterizar a los encuestados se definieron los siguientes códigos de pertenencia a la o las instituciones y a un determinado área disciplinar:
A: Institución Pública
B: Institución Privada
C: Inst. Públicas y Privadas
S: Cs. Sociales
E: Cs. Exactas
N: Cs. Naturales
H: Cs. Humanísticas

Sujeto A.H.1.

\begin{tabular}{|c|l|}
\hline Asignatura: & Filosofía \\
\hline Titulo de grado & Profesora en Ciencias de la Educación. UNNE \\
\hline Antigüedad & 1 a 5 años \\
\hline Instituciones. & Públicas periféricas \\
\hline $\begin{array}{c}\text { Estudios de } \\
\text { postítulo en la } \\
\text { Especialidad. }\end{array}$ & Curso de posgrado en instituciones, grupos y currículum \\
\hline $\begin{array}{c}\text { Estudios de } \\
\text { postítulo en } \\
\text { Educación }\end{array}$ & Curso de posgrado en instituciones, grupos y curriculum \\
\hline
\end{tabular}

Se pudo establecer la presencia de un Conocimiento académico marcado por una formación no tradicional y con rasgos reflexivos, pero a su criterio con poca relación con la realidad.

Esta situación de inicio genera en su experiencia docente fuertes incertidumbres respecto de su rol docente.

Acepta la identificación realizada en la devolución de los análisis parciales subrayando la importancia central que le brinda a los contenidos conceptuales en su diseño y desarrollo de clases, situación más cercana al modelo tradicional de enseñanza, junto con un fuerte interés en la búsqueda de estrategias innovadoras, que movilicen un proceso más independiente del sujeto que aprende. La presencia de marcadas características tradicionales y constructivistas dan cuenta de un modelo didáctico en transición, no anclado en posturas netamente absolutistas en sus concepciones epistemológicas.

Siguiendo con la teoría personal del aprendizaje, se vuelve a confirmar aspectos relacionados con concepciones que van desde la apropiación hasta la asimilación de los contenidos, estableciendo que en las instituciones en las que trabaja prácticamente sólo se produce la asimilación de contenidos, desde esta mirada el sujeto que aprende toma del exterior determinados contenidos y se entiende a la comunicación de los contenidos como proceso lineal que no sufre alteraciones. El alumno que va a aprender algo lo hace o porque no posee el contenido o porque el que posee es incorrecto. (Cf. Porlán y Rivero, 1998) 
La docente insiste en diferenciar entre un sujeto ideal formulado desde las teorías y un sujeto real muy diferente.

Las rutinas y guiones de acción reconocidas claramente por la docente y recogidas durante las observaciones, son la explicación, el interés y la evaluación de contenidos conceptuales.

No reconoce diferencias en las instituciones en las que trabaja y mantiene que en ambas el trabajo realizado fue individual ya que no hubieron mayores intercambios con colegas.

Por iniciativa propia se encuentra estableciendo relación con otro docente en la institución observada.

Resumiendo lo anterior se puede observar una leve pero creciente evolución en las concepciones docentes pertenecientes a su conocimiento profesional, una búsqueda de cambio y de soluciones a las problemáticas de su práctica.

\section{Sujeto B.S.2.}

\begin{tabular}{|c|l|}
\hline Asignatura: & Ciencias Sociales, Ciencias Políticas, Historia \\
\hline Titulo de grado & Profesora en Historia (Inst del Prof Gabriela Mistral) \\
\hline Antigüedad & 11 a 20 años \\
\hline Instituciones. & Privadas céntrica y periférica. \\
\hline $\begin{array}{c}\text { Estudios de postítulo } \\
\text { en la Especialidad. }\end{array}$ & Archivista (UNNE) \\
\hline $\begin{array}{c}\text { Estudios de postítulo } \\
\text { en Educación }\end{array}$ & $\begin{array}{l}\text { Curso de Actual. “Enseñanza de las Ciencias Sociales" FLACSO } \\
\text { Licenciada en Gestión Educativa (UNSE) }\end{array}$ \\
\hline
\end{tabular}

Se pudo observar la presencia de un Conocimiento académico marcado por una formación enciclopedista, con falta de "espíritu critico" en el estudio de los contenidos, donde la historia era enseñada desde un sin fin de hechos cronológicos. Dicho conocimiento, guiado por el interés de un conocimiento riguroso de la historia y por la investigación, se relaciona con los saberes que se fueron generando en la experiencia docente y a partir de su desarrollo profesional en instituciones públicas, al inicio de su carrera y en las dos instituciones privadas en la actualidad.

La docente puede reconocer una evolución en su conocimiento profesional y en sus prácticas docentes a partir de la búsqueda personal, de características personales que descubre desde su infancia, de perfeccionamiento disciplinar, de modelos tomados de otros docentes y de observaciones de otras prácticas. Esta evolución puede ser caracterizada desde las categorías analizadas en el cuestionario, según lo relatado la docente se pasa de un modelo didáctico tradicional de la enseñanza (marcado por su formación académica) a un modelo alternativo (a partir de la búsqueda personal y diversas experiencias profesionales) desde donde se sostiene una fuerte relativización del papel de los contenidos científicos como única fuente del conocimiento escolar. Se da mayor relevancia a los intereses de profesores y alumnos. Este modelo o tendencia intenta superar la disociación entre teoría y práctica en la enseñanza, y recuperar una 
visión de conjunto de los estructurantes del método didáctico sin caer en reduccionismos ni dicotomías. (Alcalá, 2002)

Estos dos aspectos están presentes en la realidad de sus prácticas observadas y su propio reconocimiento.

Algo similar sucede en relación con la teoría personal del aprendizaje; su formación académica relacionada en mayor medida con una teoría por asimilación, que se manifiesta en sus primeras experiencias prácticas y la actualidad fuertemente marcada por una teoría basada en la construcción del aprendizaje y en la búsqueda de una postura crítica ante el mismo. Pozo (2000) entiende a esta manera de comprender el aprendizaje como Teoría constructivista: admite la existencia de saberes múltiples, al romper la correspondencia entre conocimiento adquirido y realidad. Para que tenga lugar esa construcción es necesario que los procesos psicológicos se orienten más hacia la regulación del funcionamiento cognitivo del sujeto que hacia la mera apropiación de un conocimiento previamente establecido.

La docente confirma la importancia de la experiencia profesional como componente principal en la construcción y evolución de su conocimiento docente.

Respecto de las rutinas y guiones de acción, la docente puede reconocer como invariantes en sus prácticas el uso de determinadas estrategias de enseñanza (mapas o esquemas conceptuales) y la inclusión de determinados contenidos por preferencias personales, estas estrategias a su vez fueron identificadas en las observaciones de clase y en su relato práctico en el cuestionario.

Reconoce la existencia de adaptación por parte del docente a las instituciones en las cuales se desenvuelve, esta adaptación mencionada da cuenta de lo dicho por Gimeno Sacristán (1997: 200) en relación con los conocimientos docentes y sus contextos de influencia y desarrollo, para él tanto los contenidos como los procesos de pensamiento del docente, son fenómenos sociales desarrollados dentro del marco de un puesto de trabajo configurado por variables institucionales, sociales, políticas e históricas; la "adaptación" que mencionaba la docente se relaciona estrictamente con los condicionamientos institucionales al desarrollo profesional.

Resumiendo lo anterior se puede observar una evidente y conciente evolución en las concepciones docentes pertenecientes a su conocimiento profesional respecto de los modos de entender su disciplina y su relación con la práctica docente.

\section{Sujeto C.N.3.}

\begin{tabular}{|c|l|}
\hline Asignatura: & Ciencias Naturales - Biología \\
\hline Titulo de grado & Profesora de Biología - Licenciada en Zoología-UNNE \\
\hline Antigüedad & 6 a 10 años \\
\hline Instituciones. & Pública periférica y Privada céntrica. \\
\hline $\begin{array}{c}\text { Estudios de postítulo } \\
\text { en la Especialidad. }\end{array}$ & $\begin{array}{l}\text { Curso de enfermedades tropicales y emergentes. UCS. Entrenamiento } \\
\text { en vectores y parásitos. La Habana, Cuba. }\end{array}$ \\
\hline $\begin{array}{c}\text { Estudios de postítulo } \\
\text { en Educación }\end{array}$ & Licenciada en enseñanza de las Ciencias experimentales. \\
\hline
\end{tabular}


La docente manifestó provenir de una formación inicial con marcado peso en lo disciplinar, su Conocimiento académico está caracterizado fundamentalmente por aspectos tradicionales, sin embargo también estuvieron presentes, aunque sólo a un nivel de discurso que no pudo ser triangulado con las observaciones, matices de la teoría constructivista.

A partir de la información expuesta por la docente se puede afirmar junto con Porlán y Rivero (Op. Cit.: p.60) que los conocimientos relacionados con las Ciencias de la Educación son considerados generalmente por los docentes y en particular por ésta, como fragmentarios y descontextualizados, como "la teoría" en sentido despectivo.

La experiencia docente la llevó a buscar una punto medio en sus concepciones respecto de la enseñanza y el aprendizaje, según lo analizado en los instrumentos anteriores la docente se encuentra también en una transición entre un modelo didáctico tradicional de la enseñanza (marcado por su formación académica) a un modelo alternativo (a partir de la búsqueda personal de un equilibrio)

Esta entrevista permitió precisar aun más el estado de la transición, caracterizada o inclinada hacia modelos tradicionales de enseñanza. En estrecha relación con lo anterior se encuentra la teoría personal del aprendizaje que sustenta la docente y que viene a confirmar de alguna manera la concepción del un aprendizaje por asimilación, desde la cual se entiende que el estudiante comprende en profundidad los contenidos escolares que se le presentan desde el exterior, incorporándolos significativamente a su memoria académica. Plantea una disposición más activa del que aprende. En esta oportunidad, Pozo (2000) denomina a este tipo de teorización como teoría interpretativa: el aprendizaje es el resultado de la actividad personal del sujeto, mediante diversos procesos cognitivos (motivación, atención, aprendizaje, memoria, etc.). Sin embargo, desde el punto de vista epistemológico, se sigue asumiendo que la meta o función del aprendizaje es lograr copias lo más exactas posibles de la realidad: el aprendizaje tiene por meta imitar a la realidad, pero esto casi nunca es posible con exactitud, ya que requiere la puesta en marcha de procesos mediadores por parte del aprendiz que en muchos dominios del conocimiento hacen muy difícil, si no imposible, lograr copias exactas.

En esta ocasión, también la docente señala la importancia de la experiencia como componente principal en la construcción del conocimiento profesional.

Respecto de las rutinas y guiones de acción, puede reconocer como invariantes en sus prácticas el uso de determinadas estrategias de enseñanza como ser la explicación como intervención directa y necesaria en el proceso de enseñanza y aprendizaje, y la inclusión de determinados contenidos por prescripciones curriculares.

$\mathrm{Si}$ bien reconoce la existencia de adaptación por parte del docente en las instituciones en las cuales se desenvuelve, afirma que busca en los aprendizajes de los alumnos los mismos objetivos y no realiza modificaciones en sus estrategias didácticas. Solo reflexiona acerca del lugar del docente como proveedor de material impreso para el estudio de los contenidos en las escuelas públicas.

En ambas instituciones reconoce las importancia del intercambio de experiencias entre pares relacionadas con estrategias de resolución de problemas en el aula válidas para la práctica.

Resumiendo lo anterior se puede observar una leve evolución en las concepciones docentes pertenecientes a su conocimiento profesional respecto de los modos de entender su disciplina y su relación con la práctica docente. 
Sujeto C.E.4.

\begin{tabular}{|c|l|}
\hline Asignatura: & Matemática \\
\hline Titulo de grado & Profesora en Matemática y Cosmografía UNNE \\
\hline Antigüedad & 11 a 20 años \\
\hline Instituciones. & Pública periférica y Privada céntrica. \\
\hline $\begin{array}{c}\text { Estudios de } \\
\text { postítulo en la } \\
\text { Especialidad. }\end{array}$ & No posee \\
\hline $\begin{array}{c}\text { Estudios de } \\
\text { postítulo en } \\
\text { Educación }\end{array}$ & Sujeto del aprendizaje, Gestión, Evaluación, \\
\hline
\end{tabular}

La docente proviene de una formación inicial con una fuerte formación tradicional y caracteriza además a su Conocimiento académico por la presencia de materias pedagógicas que no brindaron elementos significativos para el análisis y la resolución de situaciones problemáticas de la realidad. Tanto esta docente como la docente anterior exponen su disconformidad con los contenidos aprendidos en esos espacios ya que no caracterizan adecuadamente al sujeto de aprendizaje "real".

En esta línea afirma que fue la experiencia docente junto con el intercambio con docentes más experimentados la que la llevaron a comprender más a sus alumnos, sobre todo en las escuelas estatales donde ella ejerció y ejerce; y entender la relaciones institucionales.

Según lo analizado en los instrumentos anteriores la docente se encuentra también en una transición entre un modelo didáctico tradicional de la enseñanza (marcado por su formación académica y sus concepciones respecto de su disciplina) a un modelo alternativo (a partir del fuerte interés por la comprensión por parte de los alumnos)

Esta entrevista permitió precisar aun más el estado de la transición, caracterizada o inclinada a los modelos tradicionales de enseñanza. La teoría personal del aprendizaje identificada tiene relación con un aprendizaje por asimilación de los contenidos, más allá de un interés manifiesto desde la entrevista por otro tipo de aprendizajes.

En consonancia con las otras docentes, la entrevistada señala la importancia de la experiencia como componente principal en la construcción del conocimiento profesional.

Respecto de las rutinas y guiones de acción, puede reconocer como invariantes en sus prácticas el uso de determinadas estrategias de enseñanza como ser la explicación por su potencial esclarecedor y como nueva implementación, las estrategias de co-aprendizaje entre pares.

Se distingue notablemente de la docente anterior al reconocer una marcada diferencia entre sus prácticas educativas en la institución pública periférica y la institución privada en la que ejerce. Manifiesta fuertes variaciones en sus prácticas, en la selección de contenidos en sus estrategias y en sus concepciones de aprendizaje. 
Una vez más los contextos institucionales a su vez condicionados socialmente, ejercen influencias en las prácticas profesionales que se desarrollan de manera diferenciada, estas prácticas diferentes se fundamentan en modos de concebir la enseñanza, el aprendizaje y la educación toda de acuerdo a los contextos sociales en los que se ejerce la docencia. Gimeno Sacristán (1997: 198) completaría de alguna manera lo antes enunciado señalando que la actividad de los profesores es una acción que transcurre dentro de una institución, y que por esa razón, su práctica está inevitablemente condicionada. La práctica docente observable es consecuencia de la modelación que realizan los profesores dentro de marcos institucionales de referencia.

Teniendo presente lo desarrollado, se está en condiciones de afirmar que las docentes que participaron de las tres fases de indagación, en sus reflexiones realizadas a partir de los análisis de los dos primeros instrumentos coinciden en tres aspectos fundamentales:

ubican en primer lugar de importancia para la construcción del conocimiento profesional docente y dentro de éste, para la conformación de sus concepciones respecto de la enseñanza y el aprendizaje, a la práctica docente;

en segundo lugar ubican como condicionante y en ocasiones determinante de sus concepciones y prácticas, al contexto institucional a su vez atravesado por las diferentes problemáticas socioculturales y económicas;

y en tercer lugar identifican a la formación inicial, diferenciando en ella la formación disciplinar respecto de la pedagógica. $Y$ argumentan en relación a esta última, la escasa significatividad para la resolución de problemas que la realidad les presenta.

\section{Conclusiones}

El trabajo de reflexión, indagación y estudio con los docentes confirmó de manera clara que es la práctica docente, la experiencia en las aulas, en las instituciones y en los diferentes contextos de la realidad las que condicionan fuertemente la construcción del Conocimiento Profesional Docente, limitando, transformando o en algunos casos resignando sus concepciones respecto de los procesos señalados. Conclusión congruente con lo señalado por Gimeno Sacristán (1997: 198) cuando sostiene que las posibilidades autónomas y competencias del profesor interaccionan dialécticamente con las condiciones de la realidad que al enseñante le vienen dadas a la hora de configurar un determinado tipo de práctica a través de la propia representación que se hace de esos condicionamientos.

La discusión de resultados que nos muestra a la formación inicial como sustento disciplinar y pedagógico con notables carencias en la formación para la práctica educativa, e insuficiente al momento de influir y esclarecer la realidad profesional nos hace suponer que esta mirada se relacionaría con concepciones ligadas a una perspectiva tecnicista desde la cual la realidad plantea problemas definidos a los que simplemente hay que aplicar el conocimiento aprendido en contexto de formación académica. Es así que el reclamo de las docentes entrevistadas apuntaban a que esa formación inicial no les daba respuestas a esa realidad, no era posible "aplicar" lo aprendido en la formación de grado para la solución de problemas de la práctica, desconociendo, al decir de Schön, que a menudo la práctica profesional se manifiesta como situaciones poco definidas, desordenadas y problemáticas desde varios ángulos, que demandan del docente la puesta en escena de soluciones creativas construidas en ese mismo contexto. (Cf. Schön, 1992) 
Sin embargo, no podemos eludir el cuestionamiento a nuestros procesos de formación inicial que son presentados como respuesta "perfecta" para los devenires de la práctica. Con esto queremos señalar que si existe en nuestros docentes esta mirada cercana a la perspectiva tecnicista, es porque determinadas concepciones o teorías sociales del conocimiento profesional se estarían sosteniendo en las carreras de formación docente.

Por esta razón, la profundización en las concepciones de los docentes a partir de las diferentes fases planteadas permite afirmar junto con Pozo (2000) que son producto de la cultura educativa en la que se han formado, con una fuerte presencia de modelos tradicionales con rasgos alternativos, de teorías que entienden al aprendizaje como asimilación de significados y de concepciones epistemológicas empiristas que subyacen a las prácticas, todas ellas en constante evolución.

Se vuelve entonces necesaria la continuidad de estudios sobre las decisiones pedagógicas contextualizadas a partir de su conocimiento didáctico del contenido, sus creencias y concepciones; su condicionamiento administrativo, curricular, institucional y social, sobre cómo es la aproximación al conocimiento didáctico de la asignatura y a las implicancias de esa aproximación para los objetivos de enseñanza y su metodología. (Cf. Llinares, 200: 42); para la promoción de un nuevo conocimiento profesional, basado en la integración de saberes académicos y experienciales, y de la puesta en marcha de enfoques formativos innovadores que lo impulsen y desarrollen. (Cf. Porlán y Rivero, 1998: 118)

Y la necesidad de futuras indagaciones en la temática, considerando que sólo su conocimiento certero permitiría: orientar el cambio de aquellas teorías implícitas de los docentes, fundamento de las concepciones y actuaciones referidas a la enseñanza y al aprendizaje; y la generación de mejores propuestas de formación de profesores, y por ende, que estas mejoras repercutan positivamente en la calidad educativa en general.

\section{Bibliografía Citada}

Alcalá, M. T. (2002) Concepciones epistemológicas de profesores de carrera de profesorados. Relaciones con la enseñanza en cátedras universitarias. Trabajo Correspondiente al período de investigación. Programa de Doctorado en Didáctica General y Didácticas Especiales. Universidad de León.

Alcalá, M. T., Fernández G. y Demuth, P. (2008) “¿Qué es el conocimiento científico para profesores del campo de las ciencias químicas que enseñan en carreras de formación docente universitarias?" en Cuadernos 34. IX Jornadas Regionales de Investigación en Humanidades y Ciencias Sociales. Facultad de Humanidades y Ciencias Sociales de la Universidad Nacional de Jujuy. ISSN: $0327-1471$.

Alliaud, A. (1998). "El maestro que aprende" en Revista Ensayos y experiencias: El maestro que aprende. Novedades Educativas. Año 4. N²3. pp.2-17

Angulo Rasco, J. (1999). De la investigación sobre la enseñanza al conocimiento docente. En A.l. Pérez Gómez, J. Barquín Ruiz y J.F. Angulo Rasco (eds.), Desarrollo Profesional del Docente. Política investigación y Práctica.. Madrid: Akal. 261-319.

Bolívar, A. (1995). El conocimiento de la enseñanza. Epistemología de la investigación curricular. Granada: Force/Universidad de Granada. 
Carter, K. (1990). “Teachers' knowledge and learning to teach" en R. Houston (ed.), Handbook of Research on Teacher Education. Nueva York: Macmillam, 291-310.

Chartier, A. M. (2000) "Fazeres ordinários da classe: uma aposta para a pesquisa e para a formação", en Revista Educaçao e Pesquisa № 26, 2, jul/dez, Brasil.

Demuth, P. y Alcalá, M. T. (2005) "El Conocimiento Profesional de los Formadores." Cuartas Jornadas de Investigación en Educación: "Nuevas configuraciones Sociales y Educación: Sujetos, Instituciones y Prácticas." Facultad de Filosofía y HumanidadesUniversidad Nacional de Córdoba. Publicación realizada en soporte óptico. ISBN $\mathrm{N}^{\circ}$ 950-33-0517-9.

Demuth, P. , Fernández, G. y Alcalá M. T. (2006) "Análisis de las concepciones didácticas y científicas de docentes del nivel polimodal de la ciudad de Corrientes". Secretaría General de Ciencia y Técnica de la Universidad Nacional del Nordeste. < http:// www. unne.edu.ar/web/cyt/cyt2006/index.htm>

Elliot, J. (1990) La investigación-acción en educación. Morata, Madrid.

Fenstenmarcher, G. D. (1994): "The knower and the known: The nature of knowledge in research on teaching" en L. DARLING-HAMMOND (Ed.). Review of Research in Education, 20, 3-56. A.E.R.A., Washington, D. C.

Fernández, G., Demuth, P. y Viña, N. (2007) "Las concepciones epistemológicas de docentes de las Ciencias Experimentales de nivel superior universitario y no universitario" Segundas Jornadas Nacionales y primeras latinoamericanas de pedagogía Universitaria. Enseñar y aprender en la universidad: "Culturas y educación en la universidad: problemas y perspectivas". Universidad Nacional de San Martín. Publicación realizada en soporte óptico. ISBN 978-987-1435-01-2.

Fernández, G., Acevedo, N. y Demuth, P. (2007) "Modelos didácticos y teorías subjetivas del aprendizaje de futuros docentes de los niveles de EGB 1 y 2 de la ciudad de Corrientes." Jornadas de Comunicación en investigación Educativa 2007. Instituto de Ciencias de la Educación de la Facultad de Humanidades UNNE. $<$ http://hum.unne.edu.ar/investigacion/educa/instituto/pdf/resumen fer.pdf.>

(Resumen)

Finocchio, S. y Pinkasz, D. (2006) "Pedagogía, Política e Intervención Crítica", en FLACSO, Especialización en Currículum y Practicas Escolares, Clase $N^{\circ} 1$.

Gimeno Sacristán, J. (1997) Docencia y cultura escolar. Reformas y modelo educativo, Buenos Aires: Ideas.

Grossman, Pamela L. (1990) The making of a teacher. Teacher knowledge and teacher education. Teachers College Press.

Huberman, M. (1983) Recipes for busy kitchens, Knowledge: Creation, Diffusion, Utilization, 4(4),pp. $478 \pm 510$.

Kennedy, Mary M. (2002) "Knowledge and Teaching" en Teachers and Teaching, 8:3, 355 370

Llinares, Salvador (2000) "Secondary School Mathematics Teacher's Professional Knowledge: a case from the teaching of the concept of function" en Teachers and Teaching, 6:1, 41 $-62$

Machiarola de Sigal, V. (1998) "Estudio sobre en pensamiento del profesor: el conocimiento práctico profesional" en Ensayos y Experiencias: El maestro que aprende. Novedades Educativas. Año 4. N²3. pp. 18-33. 
Marrero, J. (1993) "Las teorías implícitas del profesorado: vinculo entre la cultura y la práctica de enseñanza" en Rodrigo, Rodríguez y Marrero: Las teorías implícitas, un aproximación al conocimiento cotidiano. Madrid: Visor.

Montero, L. (2001) La construcción del conocimiento profesional docente. . Santa Fe: Homo Sapiens.

Porlán, R. y Rivero, A. (1998) El conocimiento de los profesores. Serie fundamentos N' 9. Colección universidad y enseñanza. Díada. Sevilla.

Pozo, J. (2000) "Concepciones de aprendizaje y cambio educativo" en Ensayos y Experiencias: $\quad$ Concepciones y prácticas en el aprendizaje y la enseñanza. Novedades Educativas. $\quad$ Año 6. $\mathrm{N}^{\circ}$ 33. pp. 4-13.

Rodrigo, M. J.; Rodríguez, A.; Marrero, J. (1993) Las teorías implícitas. Madrid. Visor.

Schön, D. (1987) La formación de profesionales reflexivos.. Barcelona. Paidós. (Ed. cast 1992)

Shulman, L. (1987) "Knowledge and Teaching: Foundations of the New Reform." en Harvard Educational Review, 57, 1, pp. 1-22. Edic. cast.: Conocimiento y enseñanza: fundamentos de las nueva reforma. Profesorado. Revista de Currículum y Formación del Profesorado, 9 (2), 2005.

Sierra Bravo, R. (1994) Técnicas de investigación Social. Teoría y Ejercicios. 9na ed. Paraninfo. 